\title{
Bioinformation
}

\author{
www.bioinformation.net
}

Views \& Challenges

\section{Artificial life and living systems: Insight into artificial life and its implications in life science research}

\author{
Sarvothaman Guruprasad and Kanagaraj Sekar* \\ Bioinformatics Centre, Supercomputer Education and Research Centre, \\ Indian Institute of Science, Bangalore - 560012, India; \\ Kanagaraj Sekar * - Email: sekar@physics.iisc.ernet.in; Phone: +91 8023601409 and +91 80 22933059; Fax: +91 80 \\ 23600085 and +918023600551 ; * Corresponding author \\ received March 12, 2006; revised March 28, 2006; accepted April 16, 2006; published online April 28, 2006
}

\begin{abstract}
:
Advanced technology has made it possible to build machines and systems like robots, which are capable of making intelligent decisions. Robots capable of self-replication and perform human functions are also available. The current challenge is to design evolutionary systems with high complexity comparable to that of biological networks. This is proposed to be achieved by ALife (Artificial Life). Here, we describe the promises provided by ALife for life sciences.
\end{abstract}

Keywords: cellular automaton; synthetic biology; artificial intelligence; artificial life

Cellular automatons and biology:

Jon Von Neumann, arguably, laid the foundations for the field of ALife. His UC (Universal Constructor) demonstrated evolutionary growth of complexity in a machine, akin to biological evolution. [1] The UC, a machine, is isolated from its own definition, analogous to the genotype and phenotype of a biological organism. The $\mathrm{UC}$ is also capable of self-replication coupled with growth in complexity. Other such and more advanced systems have also been created, some based on the UC and some not. Examples of these are the Game of Life, Tierra etc., which provide a way of studying artificial self-replication and natural selection in their own environments. [1] Appropriate mathematical conditions are to be imposed in such computer systems to eliminate trivial selfreproduction or "un-inheritable mutations", to be useful from a practical biological viewpoint.

Hence, ALife is a powerful tool for studying, simulating, and recreating biological systems and processes. The Von Neumann UC, a cellular automaton, has been a motivation for creating complex systems which are similar to biological systems. Its capability for universal computation also provides a basis for developing intelligent and complex systems which may find direct application in synthetic biology. The challenge, however, is in formalizing the notion of adaptive complexity in biology which is a grand challenge for evolutionary biology and ALife. [1]

\section{Scope of artificial life:}

ALife adopts a bottom-up methodology in designing systems or simulations that are life-like, with characteristics such as growth, death, evolution etc. That is, starting from the simplest elements and aggregating them to form complex systems. Artificial Intelligence, on the other hand looks to solve specific problems using human-like behavior, but not to build complex systems from scratch. Thus, these two 'artificial' fields are fundamentally

ISSN 0973-2063

Bioinformation 1(4): 139-140 (2006) different, each having its own professed goals. The goal of ALife is to study 'intelligence' or 'logic' underlying natural processes and using them in recreating alternative media like hardware or synthetic organs for humans. [2] With effective computation, one may be able to integrate the simulations of key components of biological networks to the level of macromolecular kinetics, which would provide further insight into biological systems. [3]

ALife not only helps simulate and understand natural processes but ALife is also aimed at building systems in the real world based on these processes. [2] ALife is already finding application in building physical devices such as evolvable hardware and robots, and in network and cyber security. The motivation has been from biological systems like the immune systems in nature. [4] More such robust systems can be designed by studying the natural and highly adaptive processes.

\section{Artificial life and synthetic biology:}

Synthetic biology is similar to the science of genetically engineered microorganisms. [5] In this respect, ALife and synthetic biology are foundationally similar. ALife is aimed at abstracting away the process of life from the natural medium and provides a novel alternative in redesigning biological systems using bottom-up methodology. Comparisons can, thus, be made between observed and predicted behavior aiding in the study of biological systems. The self replication of cellular automatons, with their similarity to biological systems, may prove to be a handy tool in designing cells and systems. This process will typically involve integrating various modeled biological components and data to form biological systems. [6]

Living systems are composed of macromolecules (DNA, RNA, proteins, carbohydrates, lipds, water and mineral salts). However, molecules performing similar functions can be designed. Synthetic biologists propose to design such molecules and systems for simulating natural 139

Bioinformation, an open access forum (c) 2006 Biomedical Informatics Publishing Group 


\section{Bioinformation}

\section{www.bioinformation.net}

\section{Views \& Challenges}

processes in living cells. [5] Building such alternative life needs effective simulation and modeling tools for study and design. The greatest challenge is the creation of standards for such designed components to allow interchangeability. An example is the Registry of Standard Biological Parts, which has been set up by the MIT team. [7] An existing, yet an exemplary standard, is the PoPS (Polymerase per Second) which is the flow of RNA polymerase over DNA and is independent of the DNA sequence. The challenge thus, is to bring an engineering approach to ALife towards the designing of biological systems.

Applied research on cost effective drug discovery systems through alternative molecules, energy generation from microorganisms and bioremediation uses fundamental evolutionary concepts in ALife. Nonetheless, a concerted effort in formalizing interdisciplinary ALife and Biology research to achieve the common goal of studying and recreating life-like and living systems is lacking. Designing living systems is non-trivial and future developments in ALife finds application in medicine and biotechnology.

\section{Conclusion:}

Artificial Life is an interdisciplinary science involving psychology, cognitive science, mathematics, biology and complex computations. Development of models in synthetic biology with appropriate validation finds enormous application in biomedicine. In its current status, standardization of synthesized biological components and formalization of methodologies remains as a challenge.

\section{References:}

[1] B. McCullin, Artificial Life, 6:347 (2000) [PMID:11348586]

[2] M. A. Bedau, Leonardo, 35: 395 (2002)

[3] T. S. Ray, Artificial Life, 1:195 (1994)

[4] R. A. Brooks, IEEE Journal of Robotics and Automation, 14 (1986)

[5] S. A. Benner \& A. M. Sismour, Nature Reviews Genetics, 6:53 (2005) [PMID:15995697]

[6] M. A. O'Malley, et al., BioEssays, 27:1270 (2005) [PMID:16299766]

[7] http://parts.mit.edu/

Edited by P. Kangueane

Citation: Guruprasad \& Sekar, Bioinformation 1(4): 139-140 (2006)

License statement: This is an open-access article, which permits unrestricted use, distribution, and reproduction in any medium, for non-commercial purposes, provided the original author and source are credited. 